\title{
Gamification and civic engagement in digital government applications: a review
}

\author{
Alysson Diniz dos Santos ${ }^{1}$, Felipe Rocha de Aquino ${ }^{1}$, \\ Lara Sucupira Furtado ${ }^{1}$, Ticiana Coelho da Silva ${ }^{1}$, Lívia Cruz ${ }^{1}$ \\ ${ }^{1}$ Insight Data Science Lab - Universidade Federal do Ceará (UFC) \\ Fortaleza - CE - Brasil \\ alyssonevirtual.ufc.br, feliperocha287@gmail.com, \\ \{lara.furtado,ticianalc, livia\}@insightlab.ufc.br
}

\begin{abstract}
In the context of Human Computer Interaction, engagement might be seen as a reflection of users' involvement and interaction with a digital system. To digital government initiatives, civic engagement refers to the ways in which citizens participate in the life of a community, in order to improve conditions for others or to help shape the community's future. Recent studies have approached civic engagement harnessed by gamification, i.e. the adoption of game elements in non-game contexts. In this context, the goal of this research is to investigate which gamification studies and features have been applied to civic engagement initiatives. This study reviews current literature, looking for the relationship between civic engagement and how it has been applied to the Human Computer Interaction context.
\end{abstract}

\section{Introduction}

The digital government landscape is continuously changing to reflect how governments are trying to find innovative digital solutions to social, economic and political issues, and how they transform themselves in the process. An important concept in current digital government applications is civic engagement, which refers to the ways in which citizens participate in the life of a community, in order to improve conditions for others or to help shape the community's future [Adler and Goggin 2005].

Engagement is also a prominent term in Human-Computer Interaction (HCI) studies, which emphasize the need to move beyond usability in order to understand and design more engaging experiences [O'Brien and Toms 2008]. Providing a higher level of engagement is one of the goals of gamification, i.e. the adoption of game elements in non-game contexts [Deterding et al. 2011]. The use of gamification is not limited to a particular discipline but is also applied to education, business, sport, health care, software, website development, and so on [Brigham 2015].

With regards to digital government solutions, leveraging gamification principles may facilitate and sustain changes in citizens' behaviour and actions. Therefore gamification might help to achieve desired policy outcomes, while increasing citizen engagement and trust [Ronzhyn et al. 2020]. Existing studies show that many of the newly available technologies and design methodologies could be used to increase active engagement in community building and civic participation [Gordon et al. 2014, Rothschild 2016]. However, only a few have investigated the role that technological design methodologies play 
in influencing participation and civic engagement [Lee and Kim 2014] and there is particularly little research on how gamification can influence and sustain community building and civic engagement on digital government[Hassan 2017].

As an emerging topic, recent publications have surveyed gamification on digital government initiatives. For instance, [Thiel 2016] finds that, although gamification might positively impact citizens' motivation, questions still remain about what specific game elements should be explored, and calls for an evaluation based also on user experience aspects, rather than on solely quantitative data. Building upon this study, in a more recent paper, [Hassan and Hamari 2020] identify generally positive outcomes for gamification in terms of civic learning, motivation and enjoyment. The authors analyze the contexts in which gamification is being utilized on digital government, and maps how it has been implemented. Both studies [Thiel 2016, Hassan and Hamari 2020] agree that researchers should adopt an understanding of gamification focused on facilitating engaging experience through the most suitable means, rather than through the classically popular means (e.g., points, and badges). Despite the interesting results, these studies lack the consideration for engagement as understood by the HCI community, and how its concepts and tools might relate to civic engagement issues.

In this context, the goal of this research is to investigate which gamification studies and features have been applied to civic engagement initiatives. To this intent, this study reviews current literature, looking for the relationship between civic engagement and how it has been applied to the HCI context. With the information extracted from the literature review, this study also aim to summarize lessons that may facilitate the success of future digital government applications.

The remainder of this paper is organized as follows: Section 2 details the methodology defined for the systematic literature review, while Section 3 presents its results. Section 4 discusses the review results, aiming at extracting lessons that may aid future digital government applications, and Section 5 presents the conclusions of this work.

\section{Systematic Literature Review Methodology}

The purpose of this systematic review is to investigate which gamification studies and features have been applied to civic engagement initiatives. We followed a systematic process that provides an objective strategy to extract information from a vast literature, since all papers examined are directly related to the study objectives. Based on the systematic process by [Caldwell and Bennett 2020], we defined the following research questions:

- RQ1: How is civic engagement conceptualized in the existing body of literature?

- RQ2: Which gamified applications have been proposed to promote civic engagement in the context of digital government?

The search process (summarized in Figure 1) occurred in January of 2021, and focused on two scientific databases, namely, ACM digital library and IEEE Explore portal. For each database, we carried out an advanced search with works that include anywhere the terms "civic engagement" AND "government" AND "gamification", with results limited to papers published between January of 2016 and December of 2020.

The initial query resulted in 368 papers ( 367 from ACM and 1 from IEEE), which were processed in order to remove repeated entries, talks, panel discussions and book 
series titles. The remaining papers were selected by reading over their title and abstract, and classified as either relevant or irrelevant, according to the following criteria: "does this paper investigate civic engagement issues?" If the answer to this question was no, then the study was excluded.

The final sample consisted of 9 peer-reviewed articles, which were further complemented with 3 studies that were found by searching for additional relevant references in each paper's bibliography list. The final 12 studies were read in order to investigate the previously mentioned research questions.

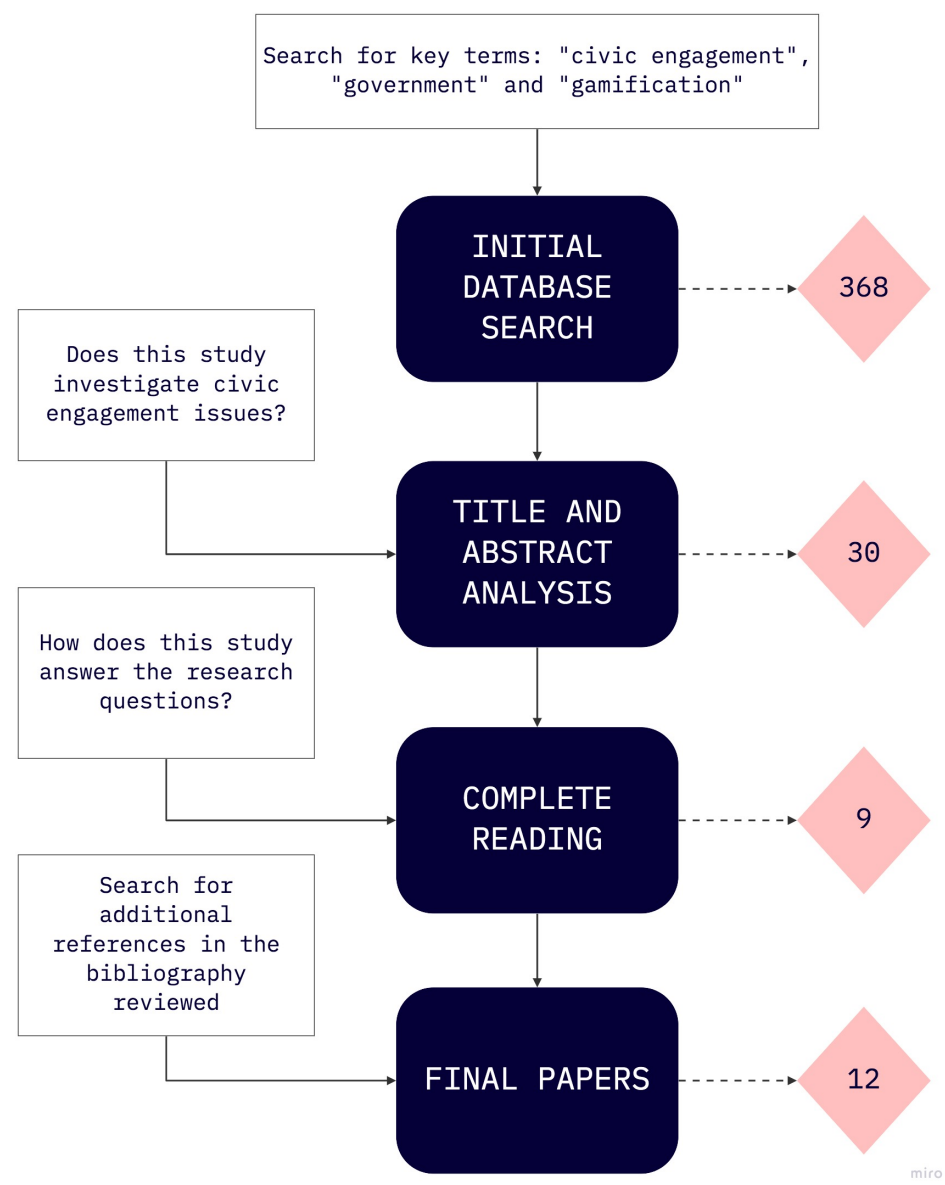

Figure 1. Systematic review steps

\section{Systematic Review Results: Gamification and Civic Engagement}

Upon analysis, it is possible to divide the identified studies into two categories: (i) theoretical studies, which involve surveys and explorations of the theoretical foundations of civic engagement, and (ii) practical applications, which detail the design and development of gamified applications for civic engagement. Those studies are synthesized in Table 1 and respectively presented in the following Sections 3.1 and 3.2.

\subsection{Theoretical studies on engagement}

As mentioned, the first set of studies discuss theoretical aspects related to civic engagement and relate to research question RQ1 (how is it civic engagement conceptualized in 
Table 1. Synopsis of reviewed papers

\begin{tabular}{|l|l|c|c|c|}
\hline ID & Reference & $\begin{array}{l}\text { Theoretical } \\
\text { study }\end{array}$ & $\begin{array}{l}\text { Practical } \\
\text { application }\end{array}$ & Evaluation \\
\hline 1 & [Palacin-Silva et al. 2018] & $\times$ & $\checkmark$ & $\checkmark$ \\
\hline 2 & [Romano et al. 2018] & $\times$ & $\checkmark$ & $\checkmark$ \\
\hline 3 & [Bianchini et al. 2016] & $\times$ & $\checkmark$ & $\checkmark$ \\
\hline 4 & [Alloghani et al. 2017] & $\checkmark$ & $\times$ & $\checkmark$ \\
\hline 5 & [Oyibo et al. 2017] & $\checkmark$ & $\times$ & $\times$ \\
\hline 6 & [Cantador and Cortés-Cediel 2018] & $\checkmark$ & $\times$ & $\times$ \\
\hline 7 & [Cortés-Cediel et al. 2018] & $\checkmark$ & $\times$ & $\times$ \\
\hline 8 & [Ronzhyn et al. 2020] & $\times$ & $\checkmark$ & $\times$ \\
\hline 9 & [Sandoval-Almazan and Valle-Cruz 2017] & $\checkmark$ & $\times$ & $\times$ \\
\hline 10 & [Thiel 2016] & $\checkmark$ & $\times$ & $\times$ \\
\hline 11 & [Hassan and Hamari 2020] & $\checkmark$ & $\times$ & $\times$ \\
\hline 12 & [Doherty and Doherty 2018] & & \\
\hline
\end{tabular}

the existing body of literature?). The discussion presented in this Section is paramount to define parameters for the analysis of the practical applications detailed in the following Sections 3.2 and 4.

As a key theoretical contribution, [Doherty and Doherty 2018] present an extensive survey on how engagement is understood by the community of HCI researchers and practitioners. According to the authors, engagement is a universal goal in the design of content, products, systems, and services. In other words, in general, every designer strives to engage users.

In the context of HCI, engagement might be seen as a reflection of users' involvement and interaction [Dobrian et al. 2011], which encompasses behavioural, emotional and cognitive components [Zyngier 2008]. These components are interwoven in a process with three distinct phases: a beginning, a period of sustention, and an end [Doherty and Doherty 2018]. This concept casts the user as both an active and receptive participant, and engagement as a continuous, synchronous process with a clearly defined beginning and end.

In turn, civic engagement refers to the ways in which citizens participate in the life of a community, in order to improve conditions for others or to help shape the community's future [Adler and Goggin 2005]. It is important to highlight that this concept attributes particular importance to the user, in this case, the citizen. Drawing a parallel with the HCI definition of engagement, the citizen plays a central active role, fundamental to the civic engagement concept. Another intersection between concepts is due to civic engagement comprising a range of individual and collective actions, as can be seen in Figure 2. Therefore, civic engagement can also be understood as a continuum of actions, similar to how engagement is viewed by HCI.

Specifically concerning the mechanics of civic engagement, [Cortés-Cediel et al. 2018] argues that an engaged citizen may feel distinct levels of energy and interest in participatory experiences, which can vary over time. To 


\title{
The Continuum of Civic Engagement
}

\author{
Informal/Private \\ Formal/Public \\ Individual Action \\ Collective Action
}



Figure 2. Civic engagement continuum [Adler and Goggin 2005].

illustrate this concept, the authors propose a life cycle that contemplates the different phases of engagement a citizen could experience. In general, these phases are: (i) the starting point of engagement, (ii) the stage of engagement, and (iii) the expiration of engagement.

The starting point of engagement demands a motivated user [Cortés-Cediel et al. 2018]. This motivation might be an intrinsic desire (the user may be personally interested in a specific governmental issue), but it also could be extrinsic (i.e. the user may need to be provoked). In the case of extrinsic motivation, for instance, it is important for the government to properly conduct a publicity campaign, in order to attract citizens of the adequate target audience. Concerning the tool itself, [Cortés-Cediel et al. 2018] argues that a careful aesthetic design is paramount for the initiative to be presented in an attractive way. Motivation can also be affected by incentives, which can be social / psychological (as social acceptance, for instance), or economic. Although economic motives may increase the quantity of "work" completed, they cannot be guaranteed to increase quality, the effects can be transitory, participants can feel manipulated and the end of the reward generally ceases the interaction [Doherty and Doherty 2018]. In addition, designers should carefully introduce the incentives, in order to avoid jeopardizing intrinsic motivation, which originates from a genuine interest and from a sense of freedom, i.e. from the lack of imposition on individual conduct [Asquer 2013].

The stage of engagement intends to ensure that citizens remain engaged with the system through elements that stimulate novelty and enable accessibility and usability [Cortés-Cediel et al. 2018]. If available, it is in this step that should be introduced participatory features, where citizens contribute by proposing ideas or actions [Cortés-Cediel et al. 2018]. This stage should also consider positive reinforcements features, such as those typically available in gamification applications [Cantador and Cortés-Cediel 2018, Ronzhyn et al. 2020]. These reinforcements pro- 
vide prompt material gratification, and potentially affect users expression of selfdetermination, allowing them to demonstrate their competence, to appreciate the sense of group membership and identification, and to contribute affirming moral and social values [Asquer 2013]. Nevertheless, it is important to highlight that an effective stage of engagement does not consist of solely adding a "layer" of game-like elements, such as goals, rules, points, badges, leaderboards, and so on, if they do not provide, on the whole, the sense of "playing a game" to the individuals [Asquer 2013]. In addition, the designer should also consider personalized feedback according to users particularities, therefore tailoring the interaction to the user needs. However, not all public services are subject to customization, mainly due to ethical or cultural reasons [Asquer 2013].

The expiration of engagement deals with the decline in the citizen interest, which generally happens naturally over time [Cortés-Cediel et al. 2018]. However, long-term engagement can develop of its own accord, where the psychological needs of users are met, meaning and value are provided, and the burden on users is low [Doherty and Doherty 2018]. Therefore, it is important for the designer to propose tools that could revitalize the engagement status, specially considering that citizens' memory about a previous interaction are critical factors to (not) participate again. Thus, in this aspect, important features are systems that provide pleasant and satisfactory experiences, and also keep citizens informed about the implementation and results of past proposals, showing them that their previous contributions were considered. The system could also employ proactive strategies, which can include mobile phone notifications, email reminders, scheduled incentives, and reward tiers [Doherty and Doherty 2018].

\subsection{Practical applications}

Another set of studies found from the review address RQ2 by detailing how gamified tools can be applied to support or enhance civic engagement, specifically in the context of digital government.

In a first example, [Palacin-Silva et al. 2018] presents an experimental study designed to understand the effects of gamification on citizen engagement in Jarvigo app (see Figure 3). The study involved the development of two versions (gamified and nongamified) of a mobile application designed to capture lake ice coverage data in Finland. The experiment involved a control group (20 participants) that interacted with the nongamified application, and an experimental group (22 participants), which received the gamified version. The evaluation measured engagement and user experience through quantitative indicators, such as: number of submissions, number of users who were active for the entire duration of the study, number of submissions per app usage, time to learn the app during first use, and others. In general, results indicated that the gamified features of the Jarvigo app increased engagement (statistically significant increase in participants' submissions), however did not affect significantly the perceived user experience.

Another gamified application is presented in [Romano et al. 2018], with the goal to involve citizens in collecting information on potential risks (such as the status of manholes, sidewalks or frozen streets) that might require emergency management actions by the government. A preliminary evaluation collected qualitative and quantitative data of 11 potential users. Though the small sample size of the study, the app was found easy to use, enjoyable, engaging and useful. 


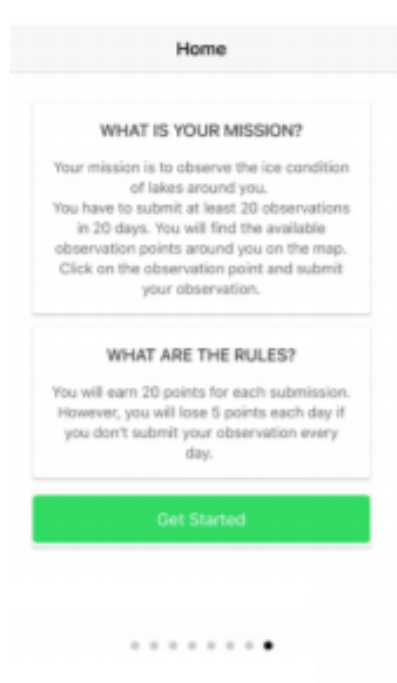

Challenge

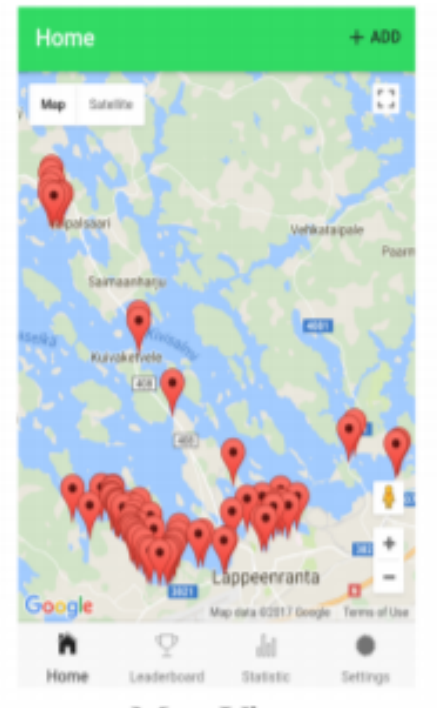

Map View

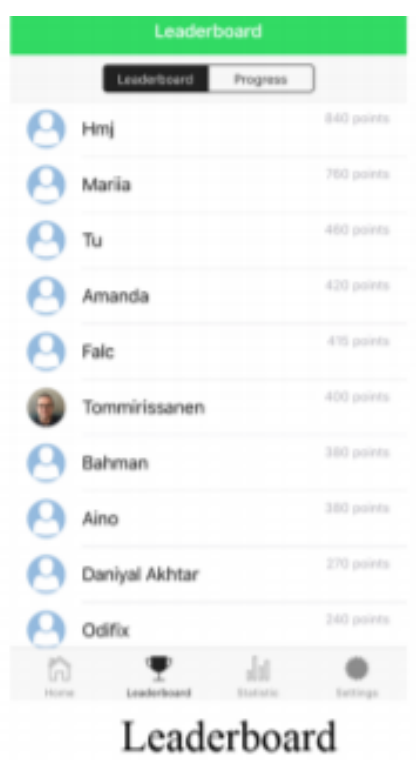

Leaderboard

Figure 3. Gamification elements in Jarvigo App [Palacin-Silva et al. 2018]

A slightly deeper approach is presented in the app TAB Sharing [Bianchini et al. 2016], in which citizens not only are able to submit a problem occurring in their community, but also are encouraged to propose a concrete and detailed description of a possible solution. The evaluation compared the two mobile versions (with and without gamification elements), collecting quantitative data of 20 volunteers. In general, the results show an increase of users' engagement in the gamified version.

In order to establish a comparison among the identified applications, it is possible to classify the gamification components employed in each of the approaches. According with [Werbach and Hunter 2012], there are 15 possible gamification components:

C1 - Achievements (defined objectives)

C2 - Avatars (visual representations of a player's character)

C3 - Badges (visual representations of achievements)

C4 - Boss Fights (especially hard challenges at the culmination of a level)

C5 - Collections (sets of items or badges to accumulate)

C6 - Combat (a defined battle, typically short-lived)

C7 - Content Unlocking (aspects available only when players reach objectives)

C8 - Gifting (opportunities to share resources with others)

C9 - Leaderboards (visual displays of player progression and achievement)

C10 - Levels (defined steps in player progression)

C11 - Points (numerical representations of game progression)

C12 - Quests (predefined challenges with objectives and rewards)

C13 - Social Graphs (representation of players' social network within the game)

C14 - Teams (defined groups of players working together for a common goal)

C15 - Virtual Goods (game assets with perceived or real-money value)

Table 2 summarizes gamification components found in the identified practical applications. For diagramming purposes, the studies are referred only trough their ID defined in the previous Table 1: 1 - [Palacin-Silva et al. 2018], 2 - [Romano et al. 2018], 3 [Bianchini et al. 2016] and 4 [Alloghani et al. 2017]. 
Table 2. Gamification components (as defined in [Werbach and Hunter 2012]) in the analyzed practical applications. Studies are identified by the IDs: 1 - [Palacin-Silva et al. 2018], 2 - [Romano et al. 2018], 3 [Bianchini et al. 2016] and 4 [Alloghani et al. 2017].

\begin{tabular}{|c|c|c|c|c|c|c|c|c|c|c|c|c|c|c|c|}
\hline ID & C1 & C2 & C3 & C4 & C5 & C6 & C7 & C8 & C9 & C10 & C11 & C12 & C13 & C14 & C15 \\
\hline 1 & $\times$ & $\checkmark$ & $\times$ & $\times$ & $\times$ & $\times$ & $\times$ & $\times$ & $\checkmark$ & $\times$ & $\checkmark$ & $\checkmark$ & $\checkmark$ & $\times$ & $\times$ \\
\hline 2 & $\checkmark$ & $\checkmark$ & $\times$ & $\times$ & $\times$ & $\times$ & $\times$ & $\times$ & $\checkmark$ & $\checkmark$ & $\checkmark$ & $\checkmark$ & $\checkmark$ & $\checkmark$ & $\times$ \\
\hline 3 & $\times$ & $\checkmark$ & $\checkmark$ & $\times$ & $\checkmark$ & $\times$ & $\checkmark$ & $\times$ & $\checkmark$ & $\checkmark$ & $\checkmark$ & $\checkmark$ & $\times$ & $\times$ & $\times$ \\
\hline 4 & $\checkmark$ & $\checkmark$ & $\checkmark$ & $\times$ & $\checkmark$ & $\times$ & $\checkmark$ & $\checkmark$ & $\checkmark$ & $\times$ & $\checkmark$ & $\checkmark$ & $\checkmark$ & $\times$ & $\times$ \\
\hline
\end{tabular}

\section{Discussion}

Considering the results of the systematic review in Section 3, it is possible to highlight some aspects regarding the most appropriate gamification components in civic engagement applications and how to evaluate them. This discussion relates with the research goals posed in Section 1, as it summarizes lessons that may facilitate the success of future digital government applications.

\subsection{Which gamification components should be employed in applications for civic engagement?}

An analysis of Table 2 informs that avatars (C2), leaderboards (C9), points (C11), quests (C12) and social graphs (C13) are the most commonly components employed in gamified applications for civic engagement. Indeed, points and leaderboards are two of the most popular components and usually represent a starting point for gamification efforts in general [Werbach and Hunter 2012]. Points and leaderboards employ mechanics of feedback and competition, highlighting player's growth and development. However, the table also presents other alternatives indicating the potential of more innovative approaches that introduce different mechanics. For instance, avatars are coupled with quests, harnessing mechanics of challenge through the proposition of puzzles or other tasks that require effort to solve. Social graphs are used in an incipient way in [Palacin-Silva et al. 2018], being possible only to observe comments made by others, while [Bianchini et al. 2016] and [Romano et al. 2018] allow players to exchange gifts or assembling in teams. These approaches are not exhaustive of the range of possible functionalities, but show a branching out from common applications of points and leaderboards.

Aside from these applications, Table 2 also reveals that there are components that were not employed by any study: boss fights (C4), combats (C6) and virtual goods (C15). We suggest that the utilization of these components could possibly enhance gamification approaches in the context of civic engagement. Through these components it could be possible, for instance, to introduce mechanics of transaction (players could be given the possibility to trade virtual goods), to simulate real-word complexities regarding choice and preferences, and to deepen competition and cooperation (with players partnering up to combat other players, or special bosses).

As stated in Section 3.1, civic engagement issues involve complex scenarios, with the interplay of multiple activities (community or political) and perspectives (such as private individual actions or public collective actions). Therefore we hope that the utilization of different gamification components might enlarge the toolbox available to systems de- 
signers, facilitating tailoring the application according to desired outcomes. This expectation is shared with [Hassan and Hamari 2020], who encourage researchers to adopt an understanding of gamification to facilitate engaging experience through the most suitable means, rather than through the classically popular means (e.g., points and leaderboards).

However, it is important to highlight that the mere introduction of different gamification components in civic engagement applications may not, by itself, issue positive results in terms of engagement [Asquer 2013]. In fact, it can even make it worse if individuals sense that the application presents inappropriate components or that the intention of the gamification intervention was to manipulate their conduct. In summary, as [Werbach and Hunter 2012] states, building an engaging gamified application takes more than checking the right boxes. It is necessary to ensure that the chosen gamification components match the particular demands of the situation.

The task of choosing appropriate gamification components for civic engagement applications might be helped by one of the many gamification design frameworks available (for a list, see [Mora et al. 2015]). Among the options, we deem that a possibly interesting one is the Octalysis framework [Chou 2019], which is based on facilitating intrinsic motivation via non-traditional components. The author stresses the need for clearly defining the intended user experience ("how do I want my users to feel?"), before choosing the gamification components. To the best of our knowledge, the application of Octalysis in the context of civic engagement applications is open for further scientific investigation.

\subsection{How to evaluate civic engagement on gamified application?}

In general, an evaluation of the gamified applications presented in Section 3.2, indicates a positive effect on civic engagement. In particular, the work from [Palacin-Silva et al. 2018] applied a rigorous evaluation, employing A/B tests with randomized control groups, which strengthen the results achieved. Despite the studies' limitations (such as the limited size of the test groups in [Palacin-Silva et al. 2018], and the absence of randomized control groups in [Romano et al. 2018, Bianchini et al. 2016]), their results seem to corroborate with the previous studies [Thiel 2016, Hassan and Hamari 2020] that gamification has the potential to influence users' engagement. However, we still observe opportunities for further improvements, in order to produce even more significant results.

At first, the studies evaluate engagement through two methods: (i) task outcomes or (ii) questionnaires. In the first, [Palacin-Silva et al. 2018] assess engagement in terms of: number of observations submitted by participants (involvement), the level of activeness of the users from beginning to end in the study (activeness) and the number of participants abandoning the study (dropout). Another task outcome approach was taken by [Bianchini et al. 2016], which collected the number of proposals, solutions, solutions details, qualified responses and activities, in order to measure engagement. The questionnaire methodology was applied by [Romano et al. 2018] to calculate the perceived engagement based on a theoretical model for technology acceptance.

There are still several possible measurements for assessing engagement. For instance, it is possible to collect subjective data through an ethnographic approach, via interviews or observational methods [Glasnapp and Brdiczka 2009]. Also, objectivityoriented approaches could be further exploited, such as automatic behaviour logging, 
which could collect and analyze data of a large number of individuals. Psychophysiological measures (as electrocardiography, heat flux, and electroencephalography) introduce an obvious computational complexity to the evaluation, but have been proven to be valuable in controlled environments through laboratory tests.

Another aspect we wish to highlight has to do with the civic engagement life cycle proposed by [Cortés-Cediel et al. 2018] and presented in Section 3.1. The evaluations detailed in [Palacin-Silva et al. 2018, Romano et al. 2018, Bianchini et al. 2016] focused only on the life cycle stage of engagement. Since the applications were tested in controlled environments, it was not possible to evaluate the success of strategies related to the starting point of engagement. Also, the studies were based on a limited time frame, precluding further conclusions about possible re-engagement processes.

In short, we believe that there is a research need for deeper and more diverse evaluation of gamified civic engagement applications. The adoption of different evaluation methods (for example, employing behaviour logging and data mining), as well as the consideration of the three stages of engagement life cycle (tackling long-term engagement studies), could issue interesting and robust results, which might not only stress the effectiveness of these applications, but also offer helpful guidelines for the design of innovative approaches.

\section{Conclusion}

This study presented a systematic literature review conducted to investigate which gamification studies and features have been applied to civic engagement initiatives. Our analysis signals a growing need for gamified applications and theoretical frameworks that consider user's motivations and engagement needs.

The discussion identified that there are gamification components (such as those identified by [Werbach and Hunter 2012]) that could be further explored in order to possibly enhance civic engagement. These components need to match the particular demands of the situation and cater to users' needs. In addition, there is a need for deeper and more diverse evaluation. The adoption of different methods, and the consideration of the whole engagement life cycle could issue interesting results.

It is worth noting that this study methodology presents limitations regarding the review procedure, the coding and the analysis since every research is prone to researcher bias and human error. The study may have failed to comprehensively identify and review relevant literature due to limits of the chosen databases and of the query keywords, thus limiting the review results. We also acknowledge the complexity of a concept such as civic engagement, which is motivated by intrinsic socio-economic and spatial factors, variables not taken into account here.

\section{Acknowledgements}

This study was funded by Projeto Plataforma Big Data para Acelerar a Transformação Digital no Estado do Ceará, with number 04772551/2020

\section{References}

Adler, R. P. and Goggin, J. (2005). What do we mean by "civic engagement"? Journal of transformative education, 3(3):236-253. 
Alloghani, M., Hussain, A., Al-Jumeily, D., Aljaaf, A., and Mustafina, J. (2017). Gamification in e-governance: Development of an online gamified system to enhance government entities services delivery and promote public's awareness. In Proceedings of the 5th International Conference on Information and Education Technology, pages $176-181$.

Asquer, A. (2013). Not just videogames: Gamification and its potential application to public services. In Digital public administration and E-government in developing nations: Policy and practice, pages 146-165. IGI Global.

Bianchini, D., Fogli, D., and Ragazzi, D. (2016). Promoting citizen participation through gamification. In Proceedings of the 9th Nordic Conference on Human-Computer Interaction, pages $1-4$.

Brigham, T. J. (2015). An introduction to gamification: adding game elements for engagement. Medical reference services quarterly, 34(4):471-480.

Caldwell, P. H. and Bennett, T. (2020). Easy guide to conducting a systematic review. ournal of Paediatrics and Child Health, 56(6):853-856.

Cantador, I. and Cortés-Cediel, M. E. (2018). Towards increasing citizen engagement in participatory budgeting digital tools. In Proceedings of the 19th Annual International Conference on Digital Government Research: Governance in the Data Age, pages 1-2.

Chou, Y.-k. (2019). Actionable gamification: Beyond points, badges, and leaderboards. Packt Publishing Ltd.

Cortés-Cediel, M. E., Gil, O., and Cantador, I. (2018). Defining the engagement life cycle in e-participation. In Proceedings of the 19th Annual International Conference on Digital Government Research: Governance in the Data Age, pages 1-2.

Deterding, S., Dixon, D., Khaled, R., and Nacke, L. (2011). From game design elements to gamefulness: defining" gamification". In Proceedings of the 15th international academic MindTrek conference: Envisioning future media environments, pages 9-15.

Dobrian, F., Sekar, V., Awan, A., Stoica, I., Joseph, D., Ganjam, A., Zhan, J., and Zhang, H. (2011). Understanding the impact of video quality on user engagement. $A C M$ SIGCOMM Computer Communication Review, 41(4):362-373.

Doherty, K. and Doherty, G. (2018). Engagement in hci: conception, theory and measurement. ACM Computing Surveys (CSUR), 51(5):1-39.

Glasnapp, J. and Brdiczka, O. (2009). A human-centered model for detecting technology engagement. In International Conference on Human-Computer Interaction, pages 621-630. Springer.

Gordon, E., Walter, S., and Suarez, P. (2014). Engagement games: A case for designing games to facilitate real-world action. Boston: EGL. Available online at http://engagementgamelab. org/pdfs/engagement-gameguide. $p d f$.

Hassan, L. (2017). Governments should play games: Towards a framework for the gamification of civic engagement platforms. Simulation \& Gaming, 48(2):249-267.

Hassan, L. and Hamari, J. (2020). Gameful civic engagement: A review of the literature on gamification of e-participation. Government Information Quarterly, 37(3):101461. 
Lee, J. and Kim, S. (2014). Active citizen e-participation in local governance: do individual social capital and e-participation management matter? In 2014 47th Hawaii International Conference on System Sciences, pages 2044-2053. IEEE.

Mora, A., Riera, D., Gonzalez, C., and Arnedo-Moreno, J. (2015). A literature review of gamification design frameworks. In 2015 7th International Conference on Games and Virtual Worlds for Serious Applications (VS-Games), pages 1-8. IEEE.

O'Brien, H. L. and Toms, E. G. (2008). What is user engagement? a conceptual framework for defining user engagement with technology. Journal of the American society for Information Science and Technology, 59(6):938-955.

Oyibo, K., Orji, R., and Vassileva, J. (2017). Investigation of the social predictors of competitive behavior and the moderating effect of culture. In Adjunct publication of the 25th conference on user modeling, adaptation and personalization, pages 419-424.

Palacin-Silva, M. V., Knutas, A., Ferrario, M. A., Porras, J., Ikonen, J., and Chea, C. (2018). The role of gamification in participatory environmental sensing: A study in the wild. In Proceedings of the 2018 CHI Conference on Human Factors in Computing Systems, pages 1-13.

Romano, M., Díaz, P., and Aedo, I. (2018). A gamified platform for civic engagement in early warning. In Proceedings of the XIX international conference on human computer interaction, pages $1-8$.

Ronzhyn, A., Wimmer, M. A., Viale Pereira, G., and Alexopoulos, C. (2020). Gamification in public service provisioning: Investigation of research needs. In The 21 st Annual International Conference on Digital Government Research, pages 294-300.

Rothschild, J. (2016). The logic of a co-operative economy and democracy 2.0: Recovering the possibilities for autonomy, creativity, solidarity, and common purpose. The Sociological Quarterly, 57(1):7-35.

Sandoval-Almazan, R. and Valle-Cruz, D. (2017). Open innovation, living labs and public officials: The case of" mapaton" in mexico. In Proceedings of the 10th International Conference on Theory and Practice of Electronic Governance, pages 260-265.

Thiel, S.-K. (2016). A review of introducing game elements to e-participation. In 2016 conference for E-democracy and open government (CeDEM), pages 3-9. IEEE.

Werbach, K. and Hunter, D. (2012). For the win: How game thinking can revolutionize your business. Wharton digital press.

Zyngier, D. (2008). (re) conceptualising student engagement: Doing education not doing time. Teaching and Teacher Education, 24(7):1765-1776. 